\title{
Piecewise Linear Representation of Finance Time Series: Quantum Mechanical Tool
}

\author{
P. CRUZ ${ }^{a}$ AND H. CRUz ${ }^{b, *}$ \\ ${ }^{a}$ U-tad, Centro Universitario de Tecnologia y Arte Digital, Universidad Camilo Jose Cela, \\ Complejo Europa Empresarial, Edificio Madrid, 28290 Las Rozas, Madrid, Spain \\ ${ }^{b}$ Departamento de Fisica and IUdEA, Universidad de La Laguna, \\ Avda. Astrofisico Francisco Sanchez, s/n, 38204 La Laguna, Tenerife, Spain
}

Doi: 10.12693/APhysPolA.138.21

*e-mail: hcruz@ull.es

\begin{abstract}
In this work, a piecewise linear representation of economic time series is presented. The representation is based on calculating local maxima and minima in time series. Then, we study the localization properties of the 1D tight-binding equation, where the on-site potential is replaced by a piecewise linear function. The piecewise linear representation is derived from economic time series databases. We carry out numerical work involving direct diagonalization to study eigenvalues of the system. This numerical scheme is applied to different segments of the time series. It is shown that this mathematical tool could be used as a moving indicator to study finance charts.
\end{abstract}

topics: Tight-binding methods (atomic physics), Econophysics, Quantum mechanics

\section{Introduction}

In the last years, theoretical frame of quantum mechanics has been applied to finance [1-5]. Mathematical formalism of quantum mechanics can provide theoretical tools to model socioeconomic time series [6]. Using this bridge, many techniques developed for quantum physics can be transferred to analyze socioeconomic data.

Ever since the pioneering work of Anderson [7], the study of quantum diffusion has been a central topic in Solid State Physics. Quantum localization is one of the most-studied topics in physics encompassing systems as disparate as solid state physics, light, sound, excitation in condensed matter physics and, most recently, cold atomic systems. For quantum diffusion, one of the oldest models is the nearest-neighbor tight-binding model (see Fig. 1),

$$
t\left(u_{n+1}+u_{n-1}\right)+V_{n} u_{n}=E_{n} u_{n}
$$

where $t$ is the hopping amplitude, $V_{n}$ is the onsite potential, $E_{n}$ is the energy eigenvalue and $u_{n}$ is the wave function amplitude at the $n$ lattice site. One well-known example of the using of (1) is $1 \mathrm{D}$ incommensurate problem. In the AubryAndré model, the on-site term is

$$
V_{n}=\lambda \cos \left(\pi \alpha n^{\nu}\right)
$$

with an incommensurate frequency. In above equation, $\alpha$ is a real number, $\lambda$ is the strength of the potential $(0 \leq \lambda \leq 2)$, and $\nu$ is taken to lie between 0 and 1 . The slow spatial variation of $V_{n}$ is crucial in producing the localization properties

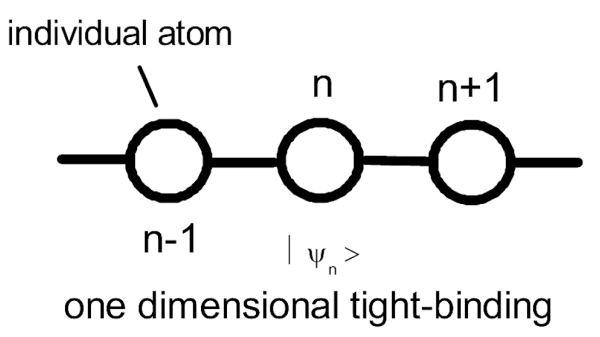

Fig. 1. Drawing of a one-dimensional atom chain. One-dimensional tight-binding model.

of the model. For $0<\nu<1$ and $0<|\lambda|<2$, it is found the existence of a mobility edge in this onedimensional model $[8,9]$. In the above potential, the case where $V_{n}=2 t$ is specially interesting: the quantum system has an eigenspectrum which forms a Cantor set in the thermodynamic limit. In such a case, all the eigenstates are extended for potential strength below a threshold value $V_{c}=2 t$ and localized above this potential energy value $V_{c}$.

In previous works, the localization properties of a 1D tight-binding model have been studied [6]. The on-site potential values were derived from financial time series and a diagonalization scheme was applied to different segments of the data. In such a model, successive differences of the natural logarithm of the price $P(n)$ have been employed to modify the incommensurate potential $V_{n}$. But because of the complex nature of financial markets, not all available data are real information, creating the problem of discerning real information from noise. 
The behavior of the Lyapunov exponent at the mobility edge was studied in [6]. This property was employed to try to forecast finance time series. However, we know that the existence of noisy financial data can limit the performance of prediction techniques on time series. The main problem is the absence of prior knowledge on what is noise and what is determinism. Taking this into account, the aim of this work will be to explore noise reduction effects in the tight-binding approach.

\section{Model}

The method is based on calculating local maxima and minima in time series. The piecewise linear representation has the advantage of being straightforward and simple. In the segmentation procedure, maxima and minima are compared with those previously found. Then, the values are obtained through an iterative process. Firstly, each data point is compared with its neighbor point and the difference between the two is calculated. Then, local maxima $x_{\max }(n)$ and minima $x_{\min }(n)$ are established, namely, $x_{\max }(n)$ and $x_{\min }(n)$ are the position in the $x$-axis of the local maximal or minimal points, respectively, and $\delta t$ is a time interval. Next, the time series are replaced by the $x_{\min }(n)$ and $x_{\max }(n)$ points after calculating local minima and maxima. In this way, a picewise linear function is obtained.

The $\delta t$ value will be used to control the degree of detail, i.e., the number of segments in the graph, to be obtained by the segmentation procedure. If $\delta t$ is increased, the degree of detail will be decreased. Figure 2 illustrates piecewise linear representations at $\delta t=10$ and $\delta t=5$. As can be seen, the number of segments in the graph (degree of detail) is increased as the time interval $\delta t$ is decreased.

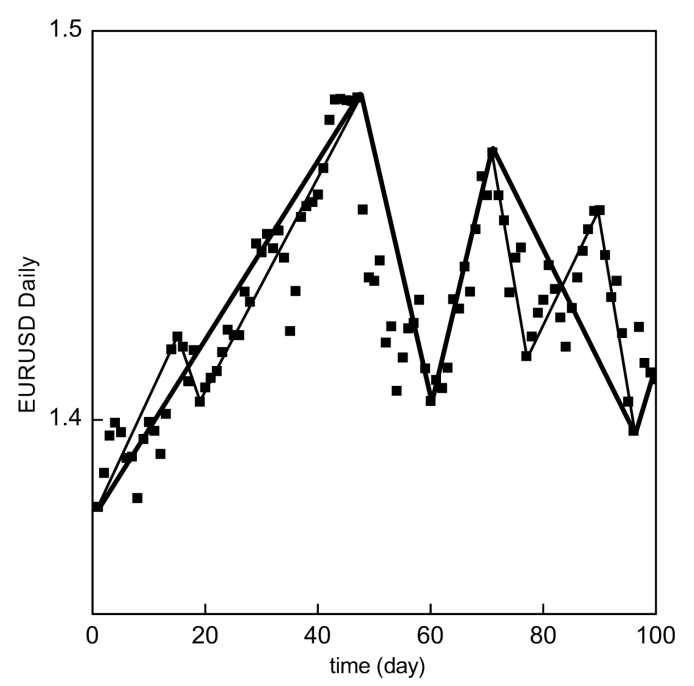

Fig. 2. Piecewise linear function and a financial time series. EURUSD Daily versus time. Thick line: $\delta t=10$. Thin line: $\delta t=5$.
In this work, an economic time series will be replaced by a piecewise linear function at a fixed $\delta t$ value. In the same manner as in [6], an on-site potential $V_{n}$ of the original model will be modified by such a piecewise linear function. As a result, the $\delta t$ parameter will be used to control noise reduction in our quantum formalism.

In addition to this, we know that the Lyapunov exponent is defined as the inverse localization length [6], i.e.,

$$
\gamma_{j}\left(E_{j}\right)=\frac{1}{N-1} \sum_{j \neq l} \ln \left|E_{j}-E_{l}\right|
$$

and the density of states as

$$
D(E)=\sum_{j} \delta\left(E-E_{j}\right)
$$

where $E_{j}$ are the eigenenergies. The $V_{n}$ potential needs to be modified in the same manner as in [6], namely

$$
V_{n}^{\prime}=\lambda \cos \left(\pi \alpha n^{\nu}\right)+\beta(n, \delta t),
$$

where

$$
\beta(n, \delta t)=\vartheta \ln \left(\frac{P(\max )}{P(\min )}\right) f(n, \delta t) .
$$

Here, $f$ is the piecewise linear function, $\vartheta$ is a coefficient, and $P(\max )$ and $P(\min )$ are the maximum and minimum price values in the time series, respectively. As can be seen in (6), if $\theta=0$ nonlinear effects vanish in our model. In order to have visible results $\theta$ needs to have a higher enough value. With no loss of generality, $\vartheta$ is taken to be $\vartheta=50$ (see $[6])$

\section{Results}

Our calculation involves direct diagonalization of the Hamiltonian to obtain the eigenenergies. This diagonalization scheme are applied to different segments of the time series. The time series is divided into $N / N_{s}$ smaller segments being $N$ the time series size. In our case, the segment size $N_{s}$ is 100 points.

In Figs. 3 and 4 we show our numerical results which clearly show the existence of a mobility edge (and a metal-insulator transition) at $E=0$. As can be seen in Figs. 3 and 4, the density of states is peaked in the middle of the energy band. In addition, the Lyapunov exponent reaches its minimum value at $E=0$. The special properties of such a potential appear when $\pi \alpha$ is equal to 0.2 and $\nu=0.7$ [8]. In this work $\pi \alpha$ is taken to be 0.2 and $\nu=0.7$.

Examining Figs. 3 and 4, we discover that we can easily obtain the slope of $\gamma(E)$,

$$
\chi(E)=\frac{\mathrm{d} \gamma(E)}{\mathrm{d} E} .
$$

Figures 5 and 6 show $\chi(t)$ (arbitrary units) versus time (day). We have also plotted the piecewise linear function and the EURUSD and USDJPY daily price, respectively.

If the price is decreased, the $\chi$ value will be increased (see arrow on Figs. 5 and 6 ) due to the $V_{n}^{\prime}$ 


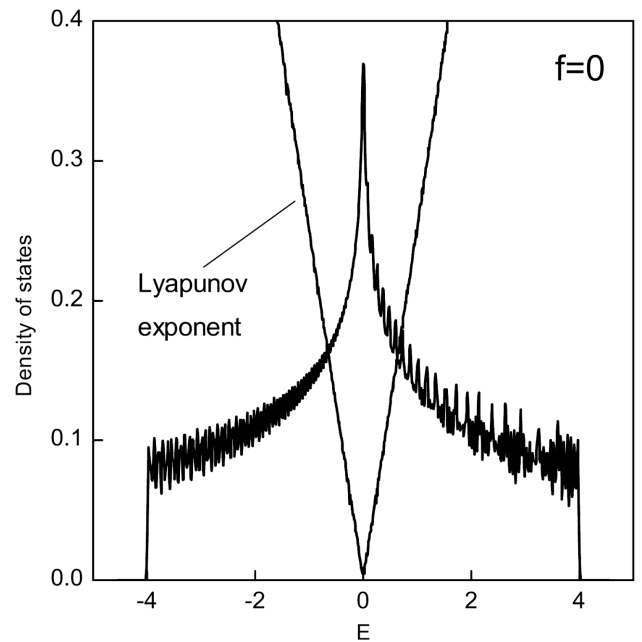

Fig. 3. Density of states and Lyapunov exponent versus energy for the potential defined in (5). We have taken $\pi \alpha=0.2, \nu=0.7$ and $f=0$. The mobility edge is at $E=0$.

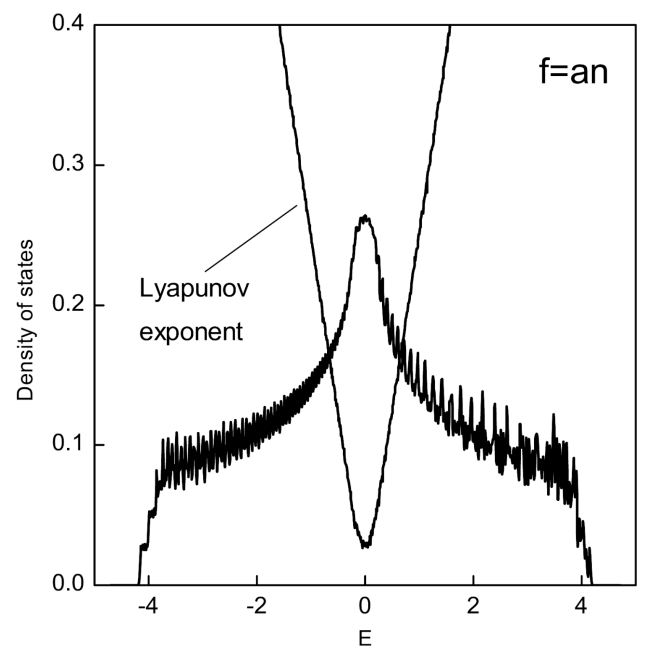

Fig. 4. Density of states and Lyapunov exponent versus energy for the potential defined in (5). We have taken $\pi \alpha=0.2, \nu=0.7, f=$ an and $a=$ 0.005 . The mobility edge is at $E=0$.

potential contribution. We have obtained a better moving indicator when a piecewise linear function is used [6]. In this way, such a mathematical tool $\chi(t)$ could be used as a moving indicator to study economic charts.

The aim of this work is to explore the use of the mathematical tools of quantum mechanics in finance. Some mathematical functions of quantum theory can have specific properties which can allow us to detect disorder effects with a high degree of sensitivity. Our attention will be paid only in the mathematical characteristics. It is clear that it makes no sense to associate physical quantities to an economic system. It has no physical meaning.

The incommensurate equation (2) employed in this work is the only known example of a onedimensional potential which allows extended and

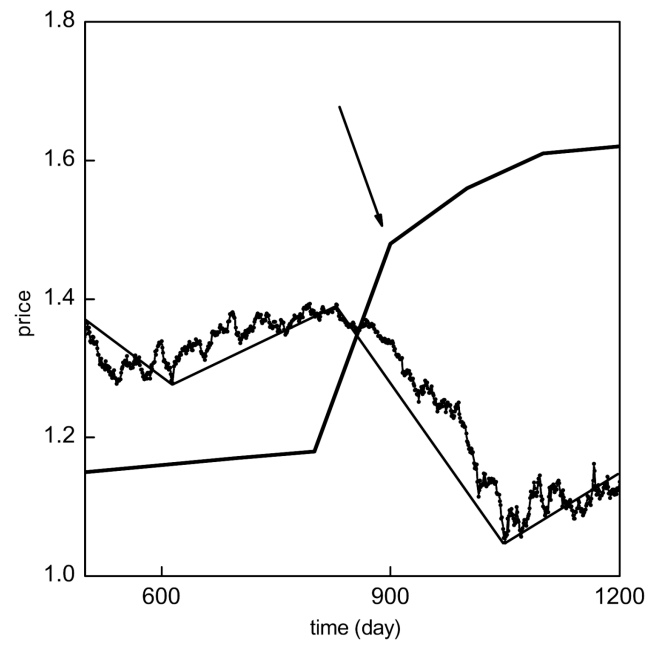

Fig. 5. Thick line: $\chi$ (arbitrary units) versus time (day). We have also plotted EURUSD daily price at $\delta t=10$. First point corresponds to daily price $2011 / 03 / 01$. Last point corresponds to daily price $2017 / 11 / 22$.

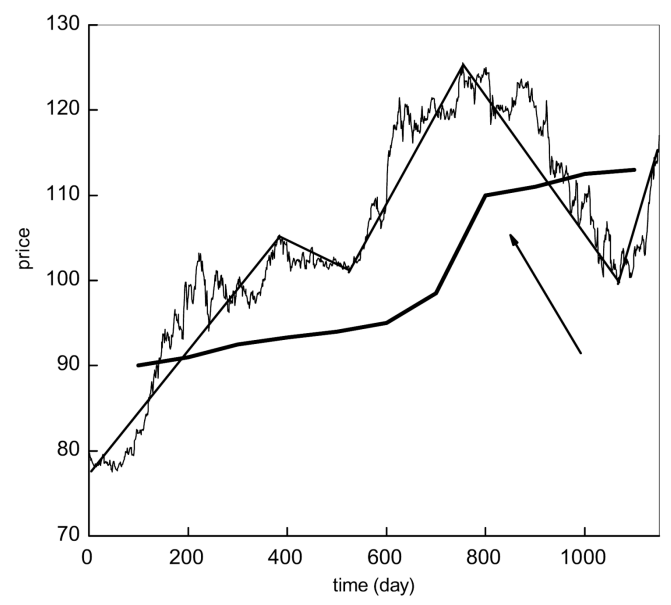

Fig. 6. Thick line: $\chi$ (arbitrary units) versus time (day). We have also plotted USDJPY daily price at $\delta t=10$. First point corresponds to daily price $2012 / 07 / 11$. Last point corresponds to daily price $2019 / 12 / 31$.

localized quantum states at the same time [8, 9]. The existence of mobility edges and a metalinsulator transition in (2) is unique. In analogy to Anderson localization of quantum waves induced by a random field, here we show that a piecewise function behavior will be reflected in the localization quantities of the potential. The piecewise linear function will be derived from our economic data.

\section{Conclusions}

In summary, we analyze the localization properties of the $1 \mathrm{D}$ tight-binding equation, where the lattice potential is derived from finance data. A diagonalization scheme is applied to different segments of 
socioeconomic time series. We have explored noise reduction effects by the using of a piecewise linear representation. We have shown the possibility of having a new moving indicator based on a quantum mechanical tool.

\section{References}

[1] R.N. Mantegna, H.E. Stanley, An Introduction to Econophysics: Correlations and Complexity in Finance, Cambridge University Press, 2000.

[2] B.E. Baaquie, Phys. Rev. E 77, 036106 (2008).

[3] B.E. Baaquie, Quantum Finance, Cambridge University Press, 2004.
[4] V. Pleron, P. Gopikrishnan, B. Rosenow, L.A. Nunes Amaral, H.E. Stanley, Phys. Rev. Lett. 83, 1471 (1999).

[5] F.G. Pedro, A. Westphal, Phys. Rev. E 95, 032144 (2017).

[6] H. Cruz, Acta Phys. Pol. A 133, 1347 (2018).

[7] P.W. Anderson, Phys. Rev. 109, 1492 (1958).

[8] S. Das Sarma, S. He, X.C. Xie, Phys. Rev. B 41, 5544 (1990).

[9] H. Cruz, S. Das Sarma, J. Phys. I France 3, 1515 (1993). 